\title{
What is an appropriate level of evidence for a digital health intervention?
}

\author{
Greaves F, Joshi I, Campbell M, Roberts S, Patel N Powell J. The Lancet. VOLUME 392, \\ ISSUE 10165, P2665-2667, DECEMBER 22, 2018
}

https://www.thelancet.com/journals/lancet/article/PIIS0140-6736(18)33129-5/fulltext

Harnessing new digital technologies to support the delivery of health services centred around the needs of patients has been embraced by the National Health Service (NHS) in England.1 Digital technologies-eg, apps, wearables, and software algorithms-have the potential to support a technology-enabled health system in which care interactions are moved away from formal settings and citizens are encouraged to manage their own health and illness. The scalability and often low marginal cost of digital interventions suggest they might deliver cost benefits to stretched services facing the demands of ageing populations living longer with higher levels of chronic disease. At the same time, a publicly funded health system has both financial and moral reasons to spend money conscientiously and judiciously to provide evidence-based effective care for its citizens.2 Some have suggested that digital medicine is different - that it does not need to be held to the established standards of evidence-based medicine in the same way that a new drug or device would. 3 The traditional comparative processes of evidence-based medicine are slow, some suggest, and struggle to keep up with the dynamic nature of digital development and delivery.4 This could stifle innovation, adoption, and growth. Robustness and rigour are seen to be at odds with rapidity and responsiveness. Others respond that there should be no digital exceptionalism and that digital tools should be held to the same standards as other healthcare technologies, including drugs and medical devices.5 In this context, the NHS in England working with the National Institute for Health and Care Excellence, Public Health England, and Med City, a publicly funded organisation that supports the growth of the life sciences ecosystem in southeast England, have developed an Evidence Standards Framework for Digital Health Technologies.6 Launched on Dec 10, 2018, this framework provides standards for the evidence of effectiveness and economic impact for digital health interventions. The framework sets the effectiveness and economic standards to help technology companies develop appropriate evidence generation plans and are complementary to regulatory and information governance standards. The framework also provides investors and evaluators, including those commissioning services at local or national level, with a clear and common understanding of what they should be looking for. We have worked with developers, clinicians, and academics to create this guidance, and have first released it in a beta form to allow for changes in response to comments and feedback. The framework is founded on a proportionate approach to risk, determined by the functionality of the digital tool. A hierarchical classification identifies the level of evidence appropriate for the intervention. For example, digital tools that provide diagnosis or treatment are in the highest level for which the minimum evidence requirement will be a high-quality experimental or quasiexperimental study with comparative data on patient outcomes. Support services and technologies designed to simply communicate information are lower in the hierarchy and the requirements are therefore designed around the credibility and accuracy of the content, including whether they meet established standards for the quality of the information 
provided. The framework also proposes some flexibility in what evidence is needed. Within each categorisation, there are both a minimum and a best practice standard. In some situations, such as when there is a high risk of death or serious injury if a product does not work, then the framework requires a move to the best practice standard with more rigorous evidence requirements. Our approach recognises that there are some things that make digital health technologies different from more traditional health-care interventions. They are often rapidly developed and tend to iterate, update, and improve, rather than provide a stable common intervention. Digital health technologies have the potential to gather and provide real-time data about use and engagement, and in some cases about outcomes. 7 These characteristics mean that it might be possible to generate both research study and real-world evidence, including data from apps and electronic health records, in a more agile fashion. The contribution of biomedical engineering and computer science academics to this area has seen new methodologies being developed, such as Multiphase Optimisation Strategy (MOST) 8 and sequential, multiple assignment, randomised trials (SMART). 9 These present opportunities for us to use faster and more efficient approaches to evidence generation. However, there should be no lowering of the quality bar and experimental or quasi-experimental studies using traditional or innovative methodologies need to conform to high standards, including transparency of methods, a-priori analysis plans, and full publication of all results. We do not believe digital health technologies to be exceptional. If money is to be spent on these interventions, they will be competing for the same scarce resources to fund them as other interventions, so they should be held to the same level of scrutiny. Beyond effectiveness, there are substantial issues of safety, information governance, and ethics that have to be considered in the assessment and use of digital technologies. These standards form part of a wider Code of Conduct for Data Driven Technologies in England, which sets out principles that industry needs to meet in these other areas, together with a set of reciprocal commitments that government will make to facilitate this code.10 Taken together, these new principles and standards are intended to create an environment that fosters creative innovation and removes barriers to adoption and uptake, while maintaining appropriate scrutiny and rigour. We hope this clarity will help digital innovators and healthcare providers to navigate this landscape. Regulators and health systems around the world are facing similar challenges, and looking to create proportionate and dynamic solutions. The proposed approach in the Evidence Standards Framework for Digital Health Technologies6 should have relevance for other jurisdictions that are also facing the dilemma of how to balance rigour and innovation. These two attributes need not stand in tension.

*Felix Greaves, Indra Joshi, Mark Campbell, Samantha Roberts, Neelam Patel, John Powell Department of Primary Care and Public Health, Imperial College London, London W6 8RP, UK (FG); Public Health England, London, UK (FG); NHS England, London, UK (IJ, SR); National Institute for Health and Care Excellence, London, UK (MC, JP); MedCity, London, UK (NP); and Nuffield Department of Primary Care Health Sciences, University of Oxford, Oxford, UK (JP) felix.greaves08@imperial.ac.uk

FG is employed by Public Health England and was part of the team that developed the evidence standards framework discussed in this Comment. IJ and SR are employed by NHS England. MC is employed by the National Institute for Health and Care Excellence (NICE) and was part of the team that developed the evidence standards framework. NP is employed by 
MedCity. JP is employed part-time by NICE and was part of the team that developed the evidence standards framework. We declare no other competing interests. This work was funded by NHS England. This Comment provides a commentary on the digital technology landscape that led to the commissioning of the evidence standards framework. The methods, concepts, and technical content of the framework document were developed by a project team at NICE under the direction of Alexia Tonnel. The key authors of the framework document to which summary reference is made in this Comment article are: Harriet Unsworth, Bernice Dillon, Lucie Collinson, Mark Salmon, Helen Powell, Mark Campbell, John Powell, and Alexia Tonnel.

1 Department of Health and Social Care. The future of healthcare: our vision for digital, data and technology in health and care. London: Department of Health and Social Care, 2018. https://www.gov.uk/government/publications/ the-future-of-healthcare-our-vision-fordigital-data-and-technology-inhealth- and-care (accessed Dec 6, 2018).

2 Sackett DL, Rosenberg WM, Gray JA, Haynes RB, Richardson WS. Evidence based medicine: what it is and what it isn't. BMJ 1996; 312: 71-72.

3 The Lancet. Is digital medicine different? Lancet 2018; 392: 95.

4 Shaywitz D. Will real world performance replace RCTs as healthcare's most important standard? Forbes, May 11, 2018. https://www.forbes.com/sites/ davidshaywitz/2018/05/11/will-real-world-performance-replace-rcts-ashealthcares- mostimportant-standard/\#660835793557 (accessed Dec 6, 2018).

5 McCartney M. Innovation without sufficient evidence is a disservice to all. BMJ 2017; 358: j3980.

6 National Institute for Health and Care Excellence. Evidence standards framework for digital health technologies. London: National Institute for Health and Care Excellence, 2018. www.nice.org.uk/digital-evidencestandards (accessed Dec 10, 2018).

7 Michie S, Yardley L, West R, Patrick K, Greaves F. Developing and evaluating digital interventions to promote behavior change in health and health care. J Med Internet Res 2017; 19: e232.

8 Collins LM, Murphy SA, Strecher V. The Multiphase Optimization Strategy (MOST) and the Sequential Multiple Assignment Randomized Trial (SMART): new methods for more potent ehealth interventions. Am J Prev Med 2007; 32: S112-18.

9 Collins LM, Nahum-Shani I, Almirall D. Optimization of behavioral dynamic treatment regimens based on the sequential, multiple assignment, randomized trial (SMART). Clin Trials 2014; 11: 426-34.

10 Department of Health and Social Care. Initial code of conduct for data-driven health and care technology. London: Department of Health and Social Care, 2018.

https://www.gov.uk/government/publications/code-of-conduct-fordata- driven-health-andcare-technology/initial-code-of-conduct-for-datadriven- health-and-care-technology (accessed Dec 5, 2018). 